Bull. Korean Math. Soc. 48 (2011), No. 1, pp. 129-140

DOI 10.4134/BKMS.2011.48.1.129

\title{
ON THE STRUCTURE OF THE FUNDAMENTAL GROUP OF MANIFOLDS WITH POSITIVE SCALAR CURVATURE
}

\author{
Jin Hong Kim and Han Chul Park
}

\begin{abstract}
The aim of this paper is to study the structure of the fundamental group of a closed oriented Riemannian manifold with positive scalar curvature. To be more precise, let $M$ be a closed oriented Riemannian manifold of dimension $n(4 \leq n \leq 7)$ with positive scalar curvature and non-trivial first Betti number, and let $\alpha$ be a non-trivial codimension one homology class in $H_{n-1}(M ; \mathbb{R})$. Then it is known as in [8] that there exists a closed embedded hypersurface $N_{\alpha}$ of $M$ representing $\alpha$ of minimum volume, compared with all other closed hypersurfaces in the homology class. Our main result is to show that the fundamental group $\pi_{1}\left(N_{\alpha}\right)$ is always virtually free. In particular, this gives rise to a new obstruction to the existence of a metric of positive scalar curvature.
\end{abstract}

\section{Introduction and statements of results}

The aim of this paper is to study the structure of the fundamental group of a closed oriented Riemannian manifold with positive scalar curvature. Since the positivity of isotropic curvature always implies that of scalar curvature, this will also be the study of the fundamental group of a Riemannian manifold with positive isotropic curvature. Indeed, our original motivation of this study came from a well-known conjecture by Gromov in [4] about the structure of the fundamental group of a Riemannian manifold with positive isotropic curvature.

To describe it more precisely, we first need to set up some notions. Most of what is presented here can be found in [9]. Let $M$ be an $n$-dimensional Riemannian manifold with the tangent bundle $T_{p} M$ at the point $p \in M$. Then the curvature operator at $p$ is the self-adjoint linear endomorphism

$$
\mathcal{R}: \Lambda^{2} T_{p} M \rightarrow \Lambda^{2} T_{p} M
$$

given by

$$
\langle\mathcal{R}(x \wedge y), u \wedge v\rangle=\langle R(x, y) u, v\rangle
$$

for $x, y, u, v \in T_{p} M$, where the inner product $\langle$,$\rangle is defined by the Riemannian$ metric on $M$ and $R$ is the Riemann curvature tensor. Next we extend the

Received May 21, 2009.

2010 Mathematics Subject Classification. 57C53.

Key words and phrases. fundamental group, positive scalar curvature. 
Riemannian metric $\langle$,$\rangle on T_{p} M$ to the complexified tangent bundle $T_{p} M \otimes \mathbb{C}$ in two ways:

(1) as a complex bilinear map, denoted by (, ).

(2) as a Hermitian inner product, denoted by $\langle\langle\rangle$,$\rangle .$

Then note that $\langle\langle z, w\rangle\rangle=(z, \bar{w})$ for $z, w \in T_{p} M \otimes \mathbb{C}$. Similarly, the Riemannian metric $\langle$,$\rangle on \Lambda^{2} T_{p} M$ can be extended in two ways to $\Lambda^{2} T_{p} M \otimes \mathbb{C}$. So we can extend the curvature operator $\mathcal{R}$ in a natural way to a complex linear map

$$
\mathcal{R}: \Lambda^{2} T_{p} M \otimes \mathbb{C} \rightarrow \Lambda^{2} T_{p} M \otimes \mathbb{C} .
$$

With these said, to each 2-plane $\sigma \subset T_{p} M \otimes \mathbb{C}$ we assign a complex sectional curvature

$$
K(\sigma)=\frac{\langle\langle\mathcal{R}(z \wedge w), z \wedge w\rangle\rangle}{\|z \wedge w\|^{2}},
$$

where $\{z, w\}$ is a basis over complex numbers for $\sigma$. Then we say that $M$ has a positive sectional curvature if $K(\sigma)>0$ for all real 2-planes $\sigma$.

An element $z \in T_{p} M \otimes \mathbb{C}$ is said to be isotropic if $(z, z)=0$, and a complex linear subspace $V \subset T_{p} M \otimes \mathbb{C}$ is totally isotropic if $z \in V$ implies $(z, z)=0$, i.e., there is no other element in $V$ except isotropic ones. We say that the curvature of a Riemannian manifold $M$ is positive on totally isotropic 2-planes or has positive isotropic curvature (PIC) if $K(\sigma)>0$ for all totally isotropic 2-planes $\sigma \subset T_{p} M \otimes \mathbb{C}$. Then the real dimension of a totally isotropic subspace of $T_{p} M$ is always less than or equal to one half of the dimension $n$ of $M$, so that this condition makes sense only for $n \geq 4$. Note also that if $\sigma$ is a 2-dimensional totally isotropic subspace of $T_{p} M \otimes \mathbb{C}$, then there is a basis $\{z, w\}$ of $\sigma$ such that

$$
z=e_{1}+i e_{2}, \quad w=e_{3}+i e_{4} \quad \text { with }\left\langle e_{i}, e_{j}\right\rangle=\delta_{i j}
$$

for $1 \leq i, j \leq 4$. Such a basis $\{z, w\}$ for $\sigma$ is called a standard basis. Since

$$
z \wedge w=\left(e_{1} \wedge e_{3}-e_{2} \wedge e_{4}\right)+i\left(e_{1} \wedge e_{4}+e_{2} \wedge e_{3}\right)
$$

we have

$$
\begin{aligned}
\langle\langle\mathcal{R}(z \wedge w), z \wedge w\rangle\rangle= & R_{1313}+R_{1414}+R_{2323}+R_{2424} \\
& -R_{1324}-R_{2413}+R_{1423}+R_{2314} \\
= & R_{1313}+R_{1414}+R_{2323}+R_{2424}+2 R_{1342}+2 R_{1423} \\
= & R_{1313}+R_{1414}+R_{2323}+R_{2424}-2 R_{1234} .
\end{aligned}
$$

Therefore the condition that a Riemannian manifold $M$ is positive on totally isotropic 2-planes says that for every orthonormal four-frames $\left\{e_{1}, e_{2}, e_{3}, e_{4}\right\}$ we have the inequality

$$
R_{1313}+R_{1414}+R_{2323}+R_{2424}-2 R_{1234}>0 .
$$

This inequality, in particular, implies the positivity of the scalar curvature (see Proposition 2.5 in [10]).

By a result of Micallef and Moore in [9], there is only one topological type of compact simply connected manifold with PIC. That is, any simply connected 
compact manifold with positive isotropic curvature is a homotopy sphere. On the other hand, there is a large class of non-simply connected manifolds with PIC. For the simplest one, one may take $S^{1} \times S^{n-1}$. Moreover, according to the work of Micallef and Wang in [10], the connected sum of manifolds with PIC also admits a metric of PIC. Thus the first fundamental group of a manifold with PIC can be very large. A group is said to be virtually free if it contains a finite index subgroup that is free.

With this said, the conjecture of Gromov in [4] that is still open can be stated as follows (see also [2] and [3]).

Conjecture 1.1. The fundamental group of a closed oriented Riemannian manifold with positive isotropic curvature is virtually free.

In the paper [6], by using the Ricci flow, Hamilton gave a complete classification of all closed oriented 4-dimensional Riemannian manifolds with positive isotropic curvature and with no essential incompressible space form. His classification, in particular, implies that the fundamental group of such a Riemannian manifold is virtually free.

Motivated by the Conjecture 1.1, we will show the following theorem in Section 4.

Theorem 1.2. Let $M$ be a closed oriented Riemannian manifold of dimension $n$ with positive scalar curvature and non-trivial first Betti number, and let $\alpha$ be a non-trivial codimension one homology class in $H_{n-1}(M ; \mathbb{R})$. Assume that there exists a closed oriented embedded hypersurface $N_{\alpha}$ of $M$ representing $\alpha$ of minimum volume, compared with all other closed hypersurfaces in the homology class. Then the fundamental group $\pi_{1}\left(N_{\alpha}\right)$ is always virtually free.

Recall that the positivity of isotropic curvature implies that of scalar curvature (e.g., see Proposition 5.2 in [10]). Moreover, it is known (e.g., see [12] and [8]) that if the dimension $n$ satisfies $4 \leq n \leq 7$, then for each $\alpha \in H_{n-1}(M ; \mathbb{R})$ there always exists a closed oriented embedded hypersurface $N_{\alpha}$ of $M$ representing $\alpha$ of minimum volume, compared with all other closed hypersurfaces in the homology class. So we have the following corollary.

Corollary 1.3. Let $M$ be a closed oriented Riemannian manifold of dimension $n(4 \leq n \leq 7)$ with positive isotropic curvature (or positive scalar curvature) and non-trivial first Betti number, and let $\alpha$ be a non-trivial codimension one homology class in $H_{n-1}(M ; \mathbb{R})$. Then there exists a closed oriented embedded hypersurface $N_{\alpha}$ of $M$ representing a such that the fundamental group $\pi_{1}\left(N_{\alpha}\right)$ is virtually free.

For example, if there is a closed embedded hypersurface $N_{\alpha}$ of $M$ as in the assumption of Theorem 1.2 whose fundamental group is $\mathbb{Z} \oplus \mathbb{Z}$, then the metric does not have positive scalar curvature. This can be compared with a result (Theorem 1.3 of [3]) by Fraser and Wolfson that the fundamental group of a compact Riemannian manifold of dimension $n \geq 4$ with positive isotropic 
curvature does not contain a subgroup isomorphic to $\mathbb{Z} \oplus \mathbb{Z}$. Since the inclusion map of $N_{\alpha}$ into $M$ is not necessarily injective on $\pi_{1}$, under certain circumstances Theorem 1.2 can be considered as a much better result than Theorem 1.3 in $[3]$.

This result can also be viewed as a strong evidence of the conjecture that any free abelian subgroup of the fundamental group of a compact Riemannian manifold of dimension $n \geq 4$ with positive isotropic curvature is cyclic. Further, let $L$ be a lens space whose fundamental group is $\mathbb{Z}_{p}$. Then $S^{1} \times L$ admits a metric of positive isotropic curvature, and this fits well with Corollary 1.3. For another concrete example, let $N$ be a closed oriented Riemannian manifold of dimension $n-1(4 \leq n \leq 7)$ whose fundamental group is not virtually free. If we consider the product manifold $M=S^{1} \times N$ of dimension $n$, then $M$ admits no metric of positive isotropic curvature (or even positive scalar curvature).

More generally, we can formulate the following corollary.

Corollary 1.4. Let $M$ be a closed oriented Riemannian manifold of dimension $n(4 \leq n \leq 7)$ with a Riemannian metric $g$ and non-trivial first Betti number. Suppose that there exists a codimension one class $\alpha \in H_{n-1}(M ; \mathbb{R})$ as in the assumption of Theorem 1.2 such that the fundamental group $\pi_{1}\left(N_{\alpha}\right)$ is not virtually free. Then $g$ cannot have a positive isotropic curvature (or even positive scalar curvature).

We organize this paper as follows. In Section 2, we define uniformly elliptic operators on a suitable Sobolev space which play an important role in the proof of Theorem 1.2. Section 3 is devoted to proving the boundedness from above of the homotopy fill radius of a closed embedded hypersurface and its coverings. In Section 4, we give a proof of Theorem 1.2 which will be based on some arguments of the paper [15].

\section{Uniformly elliptic operators}

The aim of this section is to give an uniformly elliptic operator on a closed embedded hypersurface of a Riemannian manifold with positive scalar curvature.

For this purpose, we first begin with the following theorem which is implicit in Theorem 1 of [12].

Theorem 2.1. Let $M$ be a closed oriented Riemannian manifold of dimension $n(4 \leq n \leq 7)$ with positive scalar curvature and non-trivial first Betti number, and let $\alpha$ be a non-trivial codimension one homology class in $H_{n-1}(M ; \mathbb{R})$. Then there exists a closed embedded hypersurface $N_{\alpha}$ of $M$ representing $\alpha$ such that the uniformly elliptic operator

$$
-\Delta+\frac{(n-3)}{4(n-2)} \tilde{R}
$$


defined on the Sobolev space $\mathcal{H}^{1}\left(N_{\alpha}\right)$ is self-adjoint and positive-definite with respect to the induced metric on $N_{\alpha}$, where $\Delta$ is the Laplace-Beltrami operator of $N_{\alpha}$ and $\tilde{R}$ is the scalar curvature with respect to the induced metric.

As a consequence, the first eigenvalue is strictly positive and its eigenfunction of the operator cannot change its sign.

Proof. By hypothesis, there exists a non-trivial homology class $\alpha$ of degree $n-1$. But, by geometric measure theory in [8], this codimension one homology class $\alpha$ can be represented by an orientable closed embedded hypersurface $N_{\alpha}$ of minimum volume, compared to all other closed hypersurfaces in the homology class. Here we need to use the restriction $n \leq 7$ of the dimension to achieve the regularity of $N_{\alpha}$.

For simplicity, we shall use $N$ instead of $N_{\alpha}$ from now on. Let $h_{i j}$ denote the second fundamental form of $N$ with respect to the induced metric and $e_{n}$ be the unit normal vector to $N$. Then we compute the second variation of the volume of $N$ along the direction $f e_{n}$ for a smooth function $f$ on $N$. Then the second variation is given by

$$
-\int_{N}\left(R_{n n}+\sum_{1 \leq i, j<n} h_{i j}^{2}\right) f^{2}+\int_{N}|\nabla f|^{2},
$$

which is nonnegative for all $f$ by the choice of the embedded hypersurface $N$.

Next, we use the Gauss equation between the curvature tensors $R_{i j k l}$ (resp. $\left.\tilde{R}_{i j k l}\right)$ on $M(\operatorname{resp} . N)$ given by

$$
\tilde{R}_{i j k l}-R_{i j k l}=h_{i i} h_{j j}-h_{i j}^{2}, \quad 1 \leq i, j<n .
$$

By summing the equation (2.2) over all $1 \leq i, j<n$, we obtain

$$
\sum_{1 \leq i, j<n} \tilde{R}_{i j i j}=\sum_{1 \leq i, j<n} R_{i j i j}+\left(\sum_{1 \leq i<n} h_{i i}\right)^{2}-\sum_{1 \leq i, j<n} h_{i j}^{2} .
$$

Since we have $\sum_{1 \leq i<n} h_{i i}=0$ by the minimality of $N$, it follows from $(2.3)$ that

$$
\begin{aligned}
R & =\sum_{1 \leq i, j \leq n} R_{i j i j}=2 \sum_{1 \leq i<n} R_{n i n i}+\sum_{1 \leq i, j<n} R_{i j i j} \\
& =2 R_{n n}+\tilde{R}+\sum_{1 \leq i, j<n} h_{i j}^{2},
\end{aligned}
$$

where $R$ (resp. $\tilde{R}$ ) denotes the scalar curvature of $M$ (resp. $N)$. Thus we have

$$
R_{n n}=\frac{1}{2}\left(R-\tilde{R}-\sum_{1 \leq i, j<n} h_{i j}^{2}\right) .
$$


Plugging the equation (2.4) into the inequality (2.1), it is easy to obtain

$$
\int_{N}|\nabla f|^{2} \geq \int_{N} \frac{1}{2}\left(R-\tilde{R}+\sum_{1 \leq i, j<n} h_{i j}^{2}\right) f^{2} .
$$

Since the scalar curvature $R$ of $M$ is positive, it follows from the last inequality that we have

$$
\int_{N}|\nabla f|^{2}>-\int_{N} \frac{\tilde{R}}{2} f^{2}
$$

for all smooth functions $f$. But this inequality (2.5) implies that for $\mu \geq 0$ the only solution of the equation given by

$$
\Delta f=\frac{(n-3)}{4(n-2)} \tilde{R} f+\mu f
$$

is the zero solution. Indeed, if it is not true, then by using the inequality (2.5) and the equation (2.6) we have

$$
\begin{aligned}
\int_{N}|\nabla f|^{2} & >-\int_{N} \frac{1}{2} \tilde{R} f^{2}-\frac{2 \mu(n-2)}{(n-3)} \int_{N} f^{2} \\
& =\frac{2(n-2)}{(n-3)} \int_{N}|\nabla f|^{2},
\end{aligned}
$$

which is impossible, since $n \geq 4$. Therefore, the elliptic operator $-\Delta+\frac{(n-3)}{4(n-2)} \tilde{R}$ on $N$ should be positive-definite. This completes the proof of Theorem 2.1.

Remark 2.2. Using a similar argument as in the proof of Theorem 2.1, one can show that the scalar curvature $\tilde{R}$ on $N$ can be made positive only after a suitable conformal change (see also [13] and [7]).

\section{Boundedness of homotopy fill radius}

The aim of this section is to show that the closed embedded hypersurface and all its covering spaces have the property that the homotopy fill radius is bounded from above by some uniform constant. This observation will play a crucial role in the present paper.

For the following theorems, we need to set up some definitions and notations. For some more related topics and details, refer to [15] and [13]. We first begin with the definition of the homotopy fill radius of a simple closed curve $\gamma$ in a compact Riemannian manifold $\Omega$ which bounds a disk in $\Omega$ (see [13] and [5]). To do so, set

$$
N_{\epsilon}(\gamma)=\left\{x \in \Omega \mid d_{\Omega}(x, \gamma) \leq \epsilon\right\} .
$$

We then define the homotopy fill radius of $\gamma$ by

$$
\begin{aligned}
& \pi_{1} \operatorname{fillrad}(\Omega, \gamma) \\
= & \sup \left\{\epsilon>0 \mid \operatorname{dist}_{\Omega}(\gamma, \partial \Omega)>\epsilon \text { and } \gamma \text { does not bound a disk in } N_{\epsilon}(\gamma)\right\} .
\end{aligned}
$$


If the boundary of $\Omega$ is empty, then the condition of $\operatorname{dist}_{\Omega}(\gamma, \partial \Omega)>\epsilon$ is vacuous. Then we define $\pi_{1} \operatorname{Rad}(\Omega)$, simply called the homotopy fill radius of $\Omega$, to be the supremum of the homotopy fill radius $\pi_{1} \operatorname{fillrad}(\Omega, \gamma)$ taken over all simple closed curves $\gamma$ bounding a disk in $\Omega$.

For example, if $\Omega$ is a ball of radius $r$ in $\mathbb{R}^{n}$, then $\pi_{1} \operatorname{Rad}(\Omega)=\frac{r}{2}$. On the other hand, if $\Omega$ is the sphere $S^{n-1}(r)$ of radius $r$ in $\mathbb{R}^{n}$, then $\pi_{1} \operatorname{Rad}(\Omega)=\frac{\pi r}{2}$. Similarly, if $\Omega$ is the cross product of $S^{n-1}(r)$ with an interval $(-T, T)$, then $\pi_{1} \operatorname{Rad}(\Omega)=\min \left\{\frac{\pi r}{2}, T\right\}$.

With these said, we then have the following theorem about the boundedness of the homotopy fill radius.

Theorem 3.1. Let $M$ be a closed oriented Riemannian manifold of dimension $n(4 \leq n \leq 7)$ with positive scalar curvature and non-trivial first Betti number, and let $\alpha$ be a non-trivial codimension one homology class in $H_{n-1}(M ; \mathbb{R})$. Then there exists a closed oriented embedded hypersurface $N_{\alpha}$ of $M$ representing $\alpha$ whose homotopy fill radius $\pi_{1} \operatorname{Rad}\left(N_{\alpha}\right)$ is less than or equal to $\sqrt{\frac{3}{2 \Lambda}} \pi$, where $\Lambda$ is a positive lower bound of the first eigenvalue of the self-adjoint elliptic operator

$$
-\Delta+\frac{(n-3)}{4(n-2)} \tilde{R}
$$

on $N_{\alpha}$.

Proof. It follows from Theorem 2.1 that there exists a closed embedded hypersurface $N_{\alpha}$ of $M$ representing $\alpha$ such that all the eigenvalues of the uniformly elliptic operator

$$
\mathcal{L}=-\Delta+\frac{(n-3)}{4(n-2)} \tilde{R}
$$

defined on the Sobolev space $\mathcal{H}^{1}\left(N_{\alpha}\right)$ is self-adjoint and positive-definite with respect to the induced metric on $N_{\alpha}$. Then, applying the proof of Proposition 1 of [13] (see also Theorem 4.1 of [15]) to the hypersurface $N_{\alpha}$ with the selfadjoint and positive-definite elliptic operator $\mathcal{L}$ immediately gives the estimate of the homotopy fill radius of $N_{\alpha}$.

For the sake of reader, we give a sketch of the rest of the proof, as follows. To do so, set $a=\frac{(n-3)}{4(n-2)}$ and $N=N_{\alpha}$ for simplicity. Let $f>0$ be the first eigenfunction of $\mathcal{L}=-\Delta+a \tilde{R}$ with the first eigenvalue $\lambda \geq \Lambda$. Then we have

$$
\Delta f+(\lambda-a \tilde{R}) f=0 .
$$

Let $\rho$ be any positive number less than $\pi_{1} \operatorname{Rad}(N)$, and let $\gamma$ be a curve such that $\pi_{1} \operatorname{fill} \operatorname{rad}(N, \gamma)>\rho$. Then for any disk $D$ spanning $\gamma$, define

$$
A_{f}(D)=\int_{D} f d \sigma, \quad d \sigma=\text { area form. }
$$

If $D$ is a minimizing immersed disk in $N$ with $\partial D=\gamma$ for $A_{f}(D)$, then the second variation of $A_{f}$ should be non-negative. Note that such a minimizing 
immersed disk exists in $N$, since if the manifold is closed or a covering of a closed manifold, then it always admits a smooth solution of the Plateau problem for a simple closed curve bounding a disk (see page 20 in [15]). Hence the first eigenvalue of the differential operator $L$ on $D$ given by

$$
L(\varphi)=-\left(\Delta \varphi+f^{-1} \nabla \varphi \cdot \nabla f+\left(a \tilde{R}-f^{-1} \Delta_{N} f-\frac{1}{2} K+f^{-1} \Delta f\right) \varphi\right),
$$

where $\Delta$ and $\nabla$ are taken over $D$, and $K$ is twice the Gaussian curvature of $D$ is non-negative. Now, if we plug the equation (3.1) into (3.2), then we have

$$
L(\varphi)=-\left(\Delta \varphi+f^{-1} \nabla \varphi \cdot \nabla f+\left(\lambda-\frac{1}{2} K+f^{-1} \Delta f\right) \varphi\right) .
$$

Let $g$ be a positive first eigenfunction of $L$ on $D$. Then it follows from (3.3) that $g$ satisfies

$$
-L(g)=\left(\Delta g+f^{-1} \nabla g \cdot \nabla f+g\left(\lambda-\frac{1}{2} K+f^{-1} \Delta f\right) \leq 0 .\right.
$$

Since $\pi_{1} \operatorname{fillrad}(N, \gamma)>\rho$, there exists a point $x$ in $D \cap \partial N_{\rho}(\gamma)$. Then consider the set $\mathcal{T}$ of all curves $\tau$ lying in $D$ which connect $x$ to $\gamma=\partial D$, and define the functional $\mathcal{I}(\tau)$ by

$$
\mathcal{I}(\tau)=\int_{\tau} f g d s
$$

where $d s$ is the arclength of $\tau$. If $\tau$ is a curve minimizing $\mathcal{I}(\tau)$ over the set $\mathcal{T}$, then we may assume that the length $l$ of $\gamma$ is greater than or equal to $\rho$. Furthermore, by the choice of $\tau$, the second variation of $\mathcal{I}$ at $\tau$ should be nonnegative. It then follows from (3.4) that, as in the proof of the paper [13], we have a positive function $h$ defined over the interval $[0, l]$ and

$$
\begin{aligned}
& h^{-1} h^{\prime \prime}+f^{-1} f^{\prime \prime}+g^{-1} g^{\prime \prime}+h^{-1} f^{-1} h^{\prime} f^{\prime}+h^{-1} g^{-1} h^{\prime} g^{\prime}+f^{-1} g^{-1} f^{\prime} g^{\prime} \\
& +\lambda \leq 0
\end{aligned}
$$

on $[0, l]$, where the differentiation is taken with respect to the arclength parameter $s$.

Finally, if $\phi$ is any function defined over $[0, l]$ which vanishes at two end points $s=0$ and $s=l$, then some simple computations starting from (3.5) give

$$
\lambda \int_{0}^{l} \phi^{2} d s \leq \frac{3}{2} \int_{0}^{l}\left(\phi^{\prime}\right)^{2} d s .
$$

This implies that the operator $-\frac{d^{2}}{d s^{2}}-\frac{2}{3} \lambda$ has non-negative first eigenvalue on $[0, l]$, and so we have

$$
l \leq \sqrt{\frac{3}{2 \lambda}} \pi \leq \sqrt{\frac{3}{2 \Lambda}} \pi
$$

Since $l \geq \rho$ and $\rho$ is an arbitrary number less than $\pi_{1} \operatorname{Rad}(N)$, we have $\pi_{1} \operatorname{Rad}(N) \leq \sqrt{\frac{3}{2 \Lambda}} \pi$. This completes the proof of Theorem 3.1. 
Next, if $\bar{N}_{\alpha}$ is any covering space of $N_{\alpha}$ in Theorem 3.1 equipped with the covering metric induced from $N_{\alpha}$, then, only after a suitable conformal change, $\bar{N}_{\alpha}$ will admit a metric of positive scalar curvature, and the elliptic operator

$$
\overline{\mathcal{L}}=-\Delta_{\bar{N}_{\alpha}}+\frac{(n-3)}{4(n-2)} R_{\bar{N}_{\alpha}}
$$

defined on the Sobolev space $\mathcal{H}_{0}^{1}\left(\bar{N}_{\alpha}\right)$ is also self-adjoint and positive-definite with respect to the covering metric. This can be seen by using a suitable partition of unity of $\bar{N}_{\alpha}$. More precisely, we have the following lemma.

Lemma 3.2. Let $a=\frac{(n-3)}{4(n-2)}$ and $\bar{N}=\bar{N}_{\alpha}$. Then the elliptic operator

$$
\overline{\mathcal{L}}=-\Delta_{\bar{N}}+a R_{\bar{N}}
$$

defined on the Sobolev space $\mathcal{H}_{0}^{1}(\bar{N})$ is positive-definite with respect to the covering metric.

Proof. We shall denote by $\pi$ the covering projection from $\bar{N}$ to $N$. Let $\mathcal{U}=$ $\left\{U_{i}\right\}$ be an open covering of $N$ such that $\pi^{-1}\left(U_{i}\right)$ is a disjoint union of open subsets of $\bar{N}$ each of which is mapped diffeomorphically onto $U_{i}$ by $\pi$. Let $\pi^{-1}\left(U_{i}\right)=\left\{\bar{U}_{i_{j}}\right\}$ and let $\overline{\mathcal{U}}=\left\{\pi^{-1}\left(U_{i}\right)\right\}$ be the open covering of $\bar{N}$. Then choose a partition of unity $\left\{\bar{\psi}_{i_{j}}\right\}$ subordinate to the open covering $\overline{\mathcal{U}}$.

Next take a compactly supported smooth function $\bar{f}$ on $\bar{N}$ whose support is contained in the union $\cup_{i_{j} \in \bar{J}} \bar{U}_{i_{j}}$. Here we may assume without loss of generality that the support of $\bar{f}$ is connected. We then make an index subset $\bar{J}^{\prime}$ of $\bar{J}$ by the following rule: for each $i$, if any, we take one and only one index $i_{j}$ from $\bar{J}$ for $\bar{J}^{\prime}$ in such a way that the union $\cup_{i_{j} \in \bar{J}^{\prime}} \bar{U}_{i_{j}}$ is connected. Then we can define a function $f$ on $N$ by

$$
f=\sum_{i_{j} \in \bar{J}^{\prime}}\left(\left.\pi\right|_{\bar{U}_{i_{j}}} ^{-1}\right)^{*}\left(\bar{\psi}_{i_{j}}\right) \cdot\left(\left.\pi\right|_{\bar{U}_{i_{j}}} ^{-1}\right)^{*}(\bar{f}) .
$$

Since $\left\{\bar{\psi}_{i_{j}}\right\}$ is a partition of unity subordinate to the open covering $\overline{\mathcal{U}}, f$ is well-defined and smooth.

Now suppose further that $\bar{f}$ does not vanish identically over the union $\cup_{i_{j} \in \bar{J}^{\prime}} \bar{U}_{i_{j}}$ and satisfies the elliptic differential equation

$$
\overline{\mathcal{L}}(\bar{f})=-\Delta_{\bar{N}} \bar{f}+a R_{\bar{N}} \bar{f}=-\mu \bar{f}, \mu>0 .
$$

Since $\bar{f}=\sum_{i_{j} \in \bar{J}} \bar{\psi}_{i_{j}} \bar{f}$, it follows from the pullback via the inverse of the map $\pi$ over each $\bar{U}_{i_{j}}\left(i_{j} \in \bar{J}^{\prime}\right)$ as in (3.6) and (3.7) that we have

$$
\mathcal{L}(f)=-\Delta_{N} f+a R_{N} f=-\mu f, \mu>0 .
$$

Since the elliptic operator $\mathcal{L}$ on $N$ is positive-definite, this implies that $f$ should be the zero solution. But clearly this contradicts the choice of $\bar{f}$ and so $f$. This completes the proof. 
As a consequence, if $\Lambda_{\bar{N}_{\alpha}}>0$ is a positive lower bound of the first eigenvalue of the operator, then the homotopy radius $\pi_{1} \operatorname{Rad}\left(\bar{N}_{\alpha}\right)$ is also bounded by the

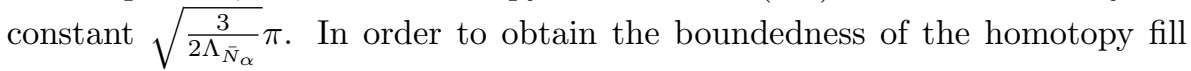
radius of $\bar{N}_{\alpha}$, it is important to notice that we do not need the existence of a metric of positive scalar curvature on $\bar{N}_{\alpha}$. Namely, we just need the existence of the uniformly elliptic operator. Hence we have the following proposition.

Proposition 3.3. Let $M$ be a closed oriented Riemannian manifold of dimension $n(4 \leq n \leq 7)$ with positive scalar curvature and non-trivial first Betti number. Let $\alpha$ be a non-trivial codimension one homology class in $H_{n-1}(M ; \mathbb{R})$. $M$ and $N_{\alpha}$ its associated closed oriented embedded hypersurface $N_{\alpha}$ of $M$ representing $\alpha$ as in Theorem 3.1. Then $N_{\alpha}$ and its covering have the property that the homotopy fill radius $\pi_{1} \mathrm{Rad}$ is bounded from above.

\section{Proof of Theorem 1.2}

The aim of this section is to give a proof of Theorem 1.2. To do so, we make use of the notion of the number of ends of a group.

Given a group $G$, the number of ends of $G$, denoted $e(G)$, can be defined by the number of geometric ends of a simplicial complex $\tilde{K}$ which is a regular covering of a finite simplicial complex $K$ and which has $G$ as the group of covering transformation. In particular, if $G$ is the first fundamental group of a closed manifold $M$ then the end $e\left(\pi_{1}(M)\right)$ of $G=\pi_{1}(M)$ is defined to be the number of ends of the universal cover $\tilde{M}$ of $M$. According to [15], the number of ends, $e(G)$, can have only $0,1,2$, or $\infty$.

We next need the notion of the accessibility of a finitely generated group $G$. We say that a finitely generated group $G$ is accessible if there is a $G$-tree $T$ such that the isotropy group $G_{e}$ of any edge $e$ of $T$ is finite and the isotropy group $G_{v}$ of any vertex $v$ of $T$ has at most one end. According to a result of Dunwoody in [1], any finitely presented group is accessible. Note that $\pi_{1}$ of a closed oriented Riemannian manifold is finitely presented. Then we need the following theorem (Theorem 5.2 in [15] and Theorem 2.4 in [11]).

Theorem 4.1. Let $N$ be a closed Riemannian manifold. Suppose that $N$ and all its covering spaces of $N$ have the property that the homotopy fill radius $\pi_{1} \mathrm{Rad}$ is bounded from above. If $G$ is a finitely generated subgroup of the fundamental group $\pi_{1}(N)$ of $N$, then $G$ cannot have exactly one end.

If we combine Theorem 4.1 with Proposition 3.3, then it is immediate to obtain the following:

Corollary 4.2. Let $M$ be a closed oriented Riemannian manifold of dimension $n(4 \leq n \leq 7)$ with positive scalar curvature and non-trivial first Betti number. Let $\alpha$ be a non-trivial codimension one homology class in $H_{n-1}(M ; \mathbb{R})$, and $N_{\alpha}$ its associated closed oriented embedded hypersurface $N_{\alpha}$ of $M$ representing $\alpha$ as in Theorem 2.1. Then the fundamental group $\pi_{1}\left(N_{\alpha}\right)$ does not have any 
finitely generated subgroups with exactly one end. In particular, $\pi_{1}\left(N_{\alpha}\right)$ does not have any subgroup isomorphic to $\pi_{1}$ of a Riemann surface.

Finally we are ready to prove a special case of the main theorem (Theorem 1.2) of this paper. The proof of Theorem 1.2 is similar and so will be left to the reader.

Theorem 4.3. Let $M$ be a closed oriented Riemannian manifold of dimension $n(4 \leq n \leq 7)$ with positive scalar curvature and non-trivial first Betti number. Let $\alpha$ be a non-trivial codimension one homology class in $H_{n-1}(M ; \mathbb{R})$, and $N_{\alpha}$ its associated closed oriented embedded hypersurface $N_{\alpha}$ of $M$ representing $\alpha$ of minimum volume, compared with all other closed hypersurfaces in the homology class. Then the fundamental group $\pi_{1}\left(N_{\alpha}\right)$ is virtually free.

Proof. For simplicity, let us denote by $N$ the closed oriented embedded hypersurface $N_{\alpha}$ of $M$ representing $\alpha$ of minimum volume, compared with all other closed hypersurfaces in the homology class. To prove the theorem, recall first that $\pi_{1}(N)$ is accessible. Note also that by Theorem 4.1 (or Theorem 5.2 of [15]) $\pi_{1}(N)$ has no finitely generated subgroups with exactly one end. Hence it follows from a result by Dunwoody in [1] that there is a $G$-tree $T$ such that the isotropy group $G_{e}$ of any edge $e$ of $T$ is finite and the isotropy group $G_{v}$ of any vertex $v$ of $T$ is finite. But this implies that by a theorem of Serre (Proposition 11 of Section II.2.6 in [14]) $\pi_{1}(N)$ is virtually free of finite rank. This completes the proof.

Acknowledgements. The author is grateful to the anonymous reader for providing valuable suggestions. This work was supported by the Korea Research Foundation Grant funded by the Korean Government (MOEHRD, Basic Research Promotion Fund) (KRF-2008-313-C00070).

\section{References}

[1] M. Dunwoody, The accessibility of finitely presented groups, Invent. Math. 81 (1985), no. 3, 449-457.

[2] A. Fraser, Fundamental groups of manifolds with positive isotropic curvature, Ann. of Math. (2) 158 (2003), no. 1, 345-354.

[3] A. Fraser and J. Wolfson, The fundamental group of manifolds of positive isotropic curvature and surface groups, Duke Math. J. 133 (2006), no. 2, 325-334.

[4] M. Gromov, Positive curvature, macroscopic dimension, spectral gaps and higher signatures, Functional analysis on the eve of the 21st century, Vol. II (New Brunswick, NJ, 1993), 1-213, Progr. Math., 132, Birkhäuser Boston, Boston, MA, 1996.

[5] M. Gromov and H. Lawson, Positive scalar curvature and the Dirac operator on complete Riemannian manifolds, Inst. Hautes Etudes Sci. Publ. Math. No. 58 (1983), 83-196.

[6] R. Hamilton, Four-manifolds with positive isotropic curvature, Comm. Anal. Geom. 5 (1997), no. 1, 1-92.

[7] J. Kazdan and F. Warner, Prescribing curvatures, Differential geometry (Proc. Sympos. Pure Math., Vol. XXVII, Stanford Univ., Stanford, Calif., 1973), Part 2, pp. 309-319. Amer. Math. Soc., Providence, R.I., 1975. 
[8] H. B. Lawson Jr., Minimal varieties in real and complex geometry, Séminaire de Mathématiques Supérieures, No. 57 (Été 1973). Les Presses de l'Université de Montréal, Montreal, Que., 1974. 100 pp.

[9] M. Micallef and J. Moore, Minimal two-spheres and the topology of manifolds with positive curvature on totally isotropic two-planes, Ann. of Math. (2) 127 (1988), no. 1, 199-227.

[10] M. Micallef and M. Wang, Metrics with nonnegative isotropic curvature, Duke Math. J. 72 (1993), no. 3, 649-672.

[11] M. Ramachandran and J. Wolfson, Fill radius and the fundamental group, Journal of Topology and Analysis 2 (2010), 99-107.

[12] R. Schoen and S. T. Yau, On the structure of manifolds with positive scalar curvature, Manuscripta Math. 28 (1979), no. 1-3, 159-183.

[13] The existence of a black hole due to condensation of matter, Comm. Math. Phys. 90 (1983), no. 4, 575-579.

[14] J.-P. Serre, Trees, Springer-Verlag, Berlin, 1980.

[15] J. Wolfson, Four manifolds with two-positive Ricci curvature, preprint (2008), arXiv: $0805.4183 \mathrm{v} 2$.

JIN HONG KIM

Department of Mathematical Sciences

Korea Advanced Institute of Science and Technology

DAEJEON 305-701, KoREA

E-mail address: jinkim@kaist.ac.kr

HAN Chul Park

Department of Mathematical Sciences

Korea Advanced Institute of Science And Technology

DAEJeOn 305-701, Korea

E-mail address: lhuntr@gmail.com 